\title{
Saúde e prevenção de acidentes infantis: um relato de experiência
}

\author{
Children's health and accident prevention: na experience report
}

Salud infantil y prevención de accidentes: un informe de experiencia

Maria Laura Verissimo Teixeira ${ }^{1 *}$, Gustavo Garcia Pacheco ${ }^{1}$, Giovanna Mendes de Oliveira ${ }^{1}$, Mariella Prini Dias Lima ${ }^{1}$, Grazielle Lima Silva ${ }^{1}$, Maíra Ferro de Sousa Touso.

\section{RESUMO}

Objetivo: Relatar a experiência de estudantes do segundo ano do curso de medicina numa atividade preventiva com pais e responsáveis numa sala de espera de puericultura em uma unidade básica de saúde em uma cidade no interior do estado de São Paulo. Relato de experiência: O presente trabalho relata a experiência de seis acadêmicos de medicina durante uma matéria da grade curricular do quarto período, na qual eles eram inseridos na atenção primária com o foco em desenvolver atividades de educação em saúde. Dentre as oficinas realizadas, encontra-se a oficina de prevenção de acidentes. Considerações finais: Os acidentes infantis são a principal causa de morte entre crianças de um a 14 anos de idade, no Brasil. Sendo que a maioria dos acidentes ocorrem na moradia e medidas simples podem evitá-los. Logo ter acesso a informação é de suma importância para a população alterar essa realidade epidemiológica. Por outro lado, as atividades desenvolvidas pelos discentes em complexidade crescente durante a graduação, através da integração ensino-serviço-comunidade, pode levar ao aperfeiçoamento da habilidade de comunicação que é de extrema importância para o contexto médico. Desse modo entende-se que atividades como essa tem um duplo impacto, ou seja, na comunidade e graduação.

Palavras-chave: Saúde, Acidente doméstico, Prevenção de acidentes.

\begin{abstract}
Objective: To report the experience of second-year medical students in a preventive activity with parents and guardians in a childcare waiting room in a basic health unit in a city in the interior of the state of São Paulo. Experience report: This paper reports the experience of six medical students during a fourth period curriculum subject, in which they were inserted in primary care with a focus on developing health education activities. Among the workshops held, there is the accident prevention workshop. Final considerations: Child accidents are the leading cause of death among children aged between one and 14 years in Brazil. Since most accidents occur at home and simple measures can prevent them. Soon, having access to information is of paramount importance for the population to change this epidemiological reality. On the other hand, the activities developed by students in increasing complexity during graduation, through the teaching-service-community integration, can lead to the improvement of communication skills, which is extremely important for the medical context. Thus, it is understood that activities like this have a double impact, that is, on the community and graduation.
\end{abstract}

Key words: Health, Domestic accident, Accident prevention.

\section{RESUMEN}

Objetivo: Informar la experiencia de estudiantes de segundo año de medicina en una actividad preventiva con padres y tutores en una sala de espera de cuidado infantil en una unidad básica de salud en una ciudad del interior del estado de São Paulo. Informe de experiencia: En este trabajo se reporta la experiencia de seis estudiantes de medicina durante una asignatura curricular de cuarto período, en la que se insertaron en

${ }^{1}$ Universidade de Franca (UNIFRAN), Franca - SP. *E-mail: marialauravteixeira@gmail.com 
la atención primaria con un enfoque en el desarrollo de actividades de educación para la salud. Entre los talleres realizados, se encuentra el taller de prevención de accidentes. Consideraciones finales: Los accidentes infantiles son la principal causa de muerte entre los niños de entre uno y 14 años en Brasil. Dado que la mayoría de los accidentes ocurren en el hogar, las medidas simples pueden prevenirlos. Pronto, tener acceso a la información es de suma importancia para que la población cambie esta realidad epidemiológica. Por otro lado, las actividades desarrolladas por los estudiantes en complejidad creciente durante la graduación, a través de la integración enseñanza-servicio-comunidad, pueden conducir a la mejora de las habilidades comunicativas, lo cual es de suma importancia para el contexto médico. Así, se entiende que actividades como esta tienen un doble impacto, es decir, en la comunidad y en la graduación.

Palabras clave: Salud, Accidentes domésticos, Prevención de accidentes.

\section{INTRODUÇÃO}

O desenvolvimento infantil é marcado por um período de amadurecimento cognitivo e descobertas por parte das crianças, para isso muitas vezes essas se submetem a atividades com possíveis riscos à saúde e seu bem-estar. Perante a essa situação cabe aos pais e responsáveis estarem atentos e preparados para supervisioná-las de modo a não subestimar seus potenciais, visto que são necessários os estímulos sensórios motores para que haja a completa maturação cerebral dessas (GURGEL AKC e MONTEIRO Al, 2016). Todavia, esses devem estar capacitados com orientações advindas do pediatra durante as consultas de puericultura, de forma que esses são preparados para diagnosticar, tratar, promover a saúde e impedir os agravos (GURGEL AKC e MONTEIRO Al, 2016; MINISTÉRIO DA SAÚDE, 2017)

O termo " acidentes" está entrando em desuso no meio acadêmico, devido a conotação trazida de imprevisto, algo não evitável e segundo o Ministério da Saúde a melhor colocação seria agravos, haja vista que expressa qualquer prejuízo à inatingibilidade física, mental e social dos indivíduos ocasionado por condições nocivas tal como acidentes, intoxicações, abuso de dogras e lesões auto ou heteroinfligidas (NASCIMENTO EN, et al, 2013; MINISTÉRIO DA SAÚDE, 2017). Dentre os principais agravos a saúde temos as injúrias não intencionais por causas externas: traumas de trânsito, queimaduras, quedas intoxicações, asfixias e agressões (GURGEL AKC e MONTEIRO AI, 2016; SENADO FEDERAL, 2017).

A fim de elucidar a temática, a Sociedade Brasileira de Pediatria (SBP) caracterizou a frequência dos acidentes domésticos, sendo esses a forma respectiva de maior ocorrência: afogamento, quedas, queimaduras e intoxicações; além de salientar sobre os maiores riscos na fase "pré-escolar" (2 a 5 anos), período com maior quantidade de relatos sobre traumas nos prontos atendimentos do país, vale salientar que o principal local de ocorrência é o ambiente doméstico, pela maior permanência, principalmente pelas crianças mais jovens (GURGEL AKC e MONTEIRO AI, 2016; CRIANÇA SEGURA BRASIL, 2020; NASCIMENTO EN, et al., 2013). Seguindo os dados do Sistema DataSUS, injúrias representam hoje a principal causa de morte de crianças de um a 14 anos no Brasil (MINISTÉRIO DA SAÚDE, 2017; BRITO MA, et al., 2017; DATASUS, 2017).

Outra questão interligada aos agravos que acometem a população pediátrica do país é o desenvolvimento econômico, de modo que o impacto atinge mediante aos custos médicos, hospitalares com internações e tratamentos, bem como a não reposição da força de trabalho num futuro próximo (NASCIMENTO EN, et al, 2013). Segundo o Sistema de Informação Hospitalares do SUS- SIH/SUS, em 2013, 140 mil pessoas nessa mesma faixa etária ( 1 a 14 anos) foram internadas devido a acidentes e todos os anos, aproximadamente 3,6 mil crianças desse intervalo de idade progridem para óbito, além do que os estudos computam e que, nos próximos anos, o número de mortes e incapacidades causadas pelos acidentes e violências acentuará cerca de 20\% e com maior repercussão em países de menor renda (GURGEL AKC e MONTEIRO Al, 2016; NASCIMENTO EN, et al, 2013; MINISTÉRIO DA SAÚDE, 2017).

Diante deste cenário, devemos saber da existência da Política Nacional de Atenção Integral à Saúde da Criança (PNAISC) (MINISTÉRIO DA SAÚDE. Portaria $n^{\circ} 1.130,2015$ ) que traduz-se por estruturar um conjunto de alçies e estratégias da rede de saúde para a prevenção de violências, acidentes e promoção da 
cultura de paz, em adição a sistematizar metodologias de suporte aos serviços especializados e procedimentos formativos para a competência da atenção à criança em condição de violência do tipo sexual, física e psicológica, negligência e/ou abandono, aspirando a instalação de linhas de cuidado na Rede de Atenção à Saúde e na rede de proteção social no território. Dessa maneira, tem-se um eixo que reflete as intervenções de prevenção para o emprego de atitudes que impulsionam a segurança das crianças que devem dar importância aos fatores de risco e vulnerabilidade e a etapa de desenvolvimento das crianças, atentando-se por tratar de um ciclo de crescimento em que a conduta exploratória é instintiva e pode colocála em cenários que representem risco à sua integridade (SOUZA RR, et al., 2019; MACÊDO VC, 2016).

Dessa forma, faz-se necessário colocar a temática em atenção social e política, enfatizando o aprimoramento dos acadêmicos e profissionais médicos com abordagens multidisciplinares, os quais tem acesso direto e constante com essa população, visto que segundo a SBP, acidentes são evitáveis na maioria dos casos, e, com medidas simples impostas pelos adultos responsáveis de modo que nessa faixa etária é necessário, abordagens passivas de proteção (GURGEL AKC e MONTEIRO Al, 2016).

Assim, esse artigo teve como objetivo relatar a experiência de estudantes do segundo ano do curso de medicina numa atividade preventiva com pais e responsáveis numa sala de espera de puericultura em uma unidade básica de saúde em uma cidade no interior do estado de São Paulo.

\section{RELATO DE EXPERIÊNCIA}

A experiência relatada foi vivenciada por seis alunos de medicina nas atividades realizadas durante o quarto período na disciplina cujo objetivo é introduzir o aluno na atenção primária de saúde. Essa disciplina é um módulo transversal presente do primeiro ao oitavo período. O módulo em questão ocorreu durante o segundo semestre de 2019 e foi composto pelo eixo temático: saúde e desenvolvimento da criança e do adolescente.

O módulo foi composto por 18 aulas, essas foram divididas em atividades práticas e teóricas. As atividades teóricas eram desenvolvidas na dependências da universidade, a metodologia de ensino nessas aulas eram mistas composta por aulas expositivas, no início que era feita com todos os alunos da turma, e depois era aplicado a metodologia de aula invertida, nessa metodologia ocorre uma inversão da lógica tradicional pois o aluno precisa fazer a internalização do conteúdo em um estudo individual, antes da aula, e no momento de encontro do grupo apenas discutir o conhecimento já adquirido e sanar possíveis dúvidas. Nesse contexto de ensino aprendizagem o professor se torna um orientador para guiar o processo de ensino.

Em contrapartida as atividades práticas, foram desenvolvidas na Unidade Básica de Saúde, essa possui uma área de abrangência que atendia cerca de 6300 pessoas por mês, esse posto de atendimento está localizado na cidade de Franca que possui 355 mil habitantes, de acordo com dados fornecidos pela Prefeitura Municipal. A cidade é situada no interior do estado de São Paulo e conta com atendimento de nível primário, secundário e terciário. Neste contexto, foram desenvolvidas atividades relacionadas com a temática: aleitamento materno, oficina de pré-natal com o grupo de gestante, exame oftalmológico na creche e aplicação de questionário sobre acidentes infantis.

A atividade de prevenção de acidentes infantis foi realizada em um único dia e participaram da atividade mães e responsáveis que estavam na sala de espera da unidade básica de saúde. Previamente, os alunos do grupo foram orientados pela preceptora a lerem um material impresso, do Ministério da Saúde, que a mesma ofertou para ampliar e consolidar tal assunto. Somado a isso, a preceptora entregou um questionário elaborado pelo projeto da Organização Não Governamental (ONG) Criança Segura Brasil para que os membros do grupo pudessem aplicá-lo nos pacientes que estavam aguardando atendimento.

A seguir, os alunos foram individualmente, conversar com as mães e responsáveis que estavam na sala de espera, aguardando consulta com a médica pediatra, onde interrogaram todos os quesitos do questionário a fim de compreender o perfil de acidentes mais prevalentes naquela população. Posteriormente, a partir dos resultados obtidos e de maneira individual, as mães e/ou responsáveis foram orientadas sobre a prevenção. Em acréscimo, a todo momento os acadêmicos tinham sua preceptora como ponto de apoio para a execução da atividade. 
O guia "ONG Criança Segura Brasil" propunha medidas simples, moderadas e difíceis de aplicar na prática. Os alunos, após acabar todas as entrevistas, fizeram uma palestra com todos os pais e responsáveis que responderam o questionário e tiveram o cuidado de repassar todas as informações. Porém sempre focando nas ações que mais se adequam a realidade da família atendida. Algumas das mudanças de fácil aplicação e que se enquadram no contexto socioeconômico dessa população foi: retirar cadeiras, bancos e camas próximo de janelas; manter a tampa da privada sempre fechada; use as bocas de trás do fogão; colocar os cabos das panelas para dentro; guardar baldes esvaziados e de cabeça para baixo longe do alcance de crianças; na garagem, ao manobrar o carro certifique-se que não há crianças por perto.

Como consequência da ação na unidade de saúde, os alunos tomaram conhecimento de diversos episódios de acidentes domiciliares que poderiam ser evitados com medidas simples de segurança. E que muitas vezes, os pais por não terem acesso a informação, não conseguiam prevenir que seus filhos se envolvessem em acidentes domésticos.

A principal dificuldade encontrada pelo grupo de alunos foi o anseio em ter a máxima compreensão do guia "ONG Criança Segura Brasil", material teórico utilizado para embasar a conduta dos acadêmicos de educação em saúde relacionada às maneiras de prevenção de acidentes infantis. Somado a isso, havia o receio de como abordar as mães e responsáveis sobre um assunto tão delicado. Ademais, foi possível notar que estes se sentiam, na maioria das vezes, constrangidos ao responder que seu filho (a) tinha passado por alguma situação de acidente doméstico. Pois havia o receio de serem julgados como culpados, pelos estudantes. Por isso, foi fundamental aos discentes a prática de um diálogo empático e acolhedor, desprovido de julgamentos.

A participação dos alunos foi fundamental nessa ação, pois a partir da mesma foi possível identificar os acidentes mais prevalentes naquela região e informar à população que tais acidentes são comuns, que as mães e responsáveis não precisavam carregar em si sentimento de culpa e que, principalmente, mudanças simples nos ambientes domésticos poderiam alterar essa realidade, prevenindo os acidentes infantis.

\section{DISCUSSÃO}

Na infância e parte da adolescência, a maioria dos acidentes ocorrem na moradia ou em regiões próximas. No Brasil, acidentes e violência foram responsáveis por 158.657 óbitos em 2017, segundo o Datasus (2017). Além disso, estima-se que existam de dez a vinte crianças e adolescentes vítimas de traumas que não são registrados para cada óbito (MINISTÉRIO DOS DIREITOS HUMANOS, 2020). Para se ter uma ideia, acidentes representam a principal causa de morte entre crianças de um a 14 anos de idade no Brasil. $O$ fato mais chocante é que a maioria desses acidentes podem ser evitados com medidas de cuidados simples (GAMA MACG, et al., 2020; ROMA KMS, et al. 2018)

$\mathrm{Na}$ atividade em questão a prevenção, isto é, as intervenções orientadas a evitar o surgimento de doenças específicas, reduzindo sua incidência e prevalência nas populações, correspondeu a identificar se já houve acidentes, como eles ocorreram, se era possível ser evitado, com qual idade ocorreu, quem estava presente e se houve consequências na criança acidentada, e posteriormente orientar os entrevistados maneiras simples e eficientes que poderiam evitar acidentes domésticos como os ocorridos (DEPALLENS MA, et al., 2020; ANDRADE AMS, et al., 2018)

Sabe-se que qualquer criança está vulnerável à se envolver em um acidente doméstico, porém de acordo com dados da Organização Mundial da Saúde (OMS) as crianças que se encontram em vulnerabilidade econômica são ainda mais propensas a se envolverem nesses acidentes, pois normalmente estão inseridas em um contexto com fatores de risco predisponentes como: infraestrutura inadequada nos espaços de lazer, creches e escolas (ORGANIZAÇÃO MUNDIAL DE SAÚDE, 2016). Além disso, fatores sociais como mãe solteira, baixo nível de formação materna, habitação precária ou família numerosas também se associam com o aumento de risco de acidentes domésticos (MINISTÉRIO DA SAÚDE, 2017). Assim verifica-se a importância de ações como as desenvolvidas pelos alunos de medicina nessa comunidade, uma vez que a maior parte da população que recebeu orientação possuía fatores de riscos sociais e ligados à infraestrutura (REDE NACIONAL PRIMEIRA INFÂNCIA, 2020). 
De outro ponto de vista, pode-se perceber por meio da atividade proposta, que foi possível ressaltar a importância da prevenção, promoção e proteção de saúde realizada por meio de acadêmicos da faculdade de medicina e até mesmo dos médicos já formados, através de uma boa relação com os entrevistados e sem julgamentos pelos ocorridos. Além disso, foi possível reforçar os conhecimentos obtidos por meio das aulas teóricas do curso e pelos materiais ofertados para os desenvolvedores da atividade em questão e, também, perceber qual ponto da matéria não havia sido fixado pelos estudantes. Portanto, foi reafirmado que a inserção do aluno em contextos reais da prática médica, em complexidade crescente durante a graduação, através da integração ensino-serviço-comunidade, pode levar ao aperfeiçoamento da habilidade de comunicação que é de extrema importância para o contexto médico (BRANDÃO ERM, et al., 2013).

Tendo em vista que a saúde consiste em viver com qualidade e que varia de acordo com a região, cultura, valores sociais e valores históricos, e que deve ser adaptada de acordo com as diferentes realidades, a comunicação é a principal ferramenta para identificar e posteriormente prevenir as possíveis consequências. Portanto é exigido dos alunos que sejam acessíveis e devem manter a confidencialidade das informações a eles confiadas, na interação com outros profissionais de saúde e o público geral, de acordo com o Artigo $n^{\circ} 4$ das Diretrizes Curriculares Nacionais do Curso de Graduação (MENDES R, et al., 2016; MINISTÉRIO DA EDUCAÇÃO, 2014).

A relação do estudante de medicina com a unidade básica de saúde é benéfica para ambas as partes, pois ao mesmo tempo no qual o aluno ganha confiança, adquire conhecimentos práticos e busca 0 desenvolvimento do vínculo médico-paciente, a comunidade recebe informações científicas de forma didática, podendo transformar o microambiente e alterar os dados epidemiológicos da região, neste caso, evitar tragédias acidentais com as crianças e adolescentes no ambiente doméstico. Assim, os coordenadores das escolas médicas e os coordenadores da atenção primária de saúde dos municípios, devem ser incentivados a promover o máximo de atividades práticas para inserir o aluno no dia-a-dia do serviço.

\section{AGRADECIMENTOS}

Agradecemos a Gislaine Cristhina Bellusse por supervisionar a atividade teórica e prática e colaborar com a aprendizagem dos alunos sobre o tema que foi digno de uma publicação.

\section{REFERÊNCIAS}

1. ANDRADE AMS, et al. Educação em saúde para prevenção de acidentes domésticos na infância.Revista de Trabalhos Acadêmicos - Universo Salvador, 2018. 25: 5-17

2. BRANDÃO ERM, et al. Práticas de Integração Ensino-Serviço-Comunidade: Reorientando a Formação Médica. Revista Brasileira de Educação Médica, 2013. 5: 2-4

3. BRITO MA, et al. Fatores de risco no ambiente doméstico para quedas em crianças menores de cinco anos. Revista Gaúcha de Enfermagem, 2017:1-9.

4. CRIANÇA SEGURA BRASIL. 2020. Disponível em: https://criancasegura.org.br/entenda-os-acidentes/. Acessado em 1 de julho de 2020.

5. DEPALLENS MA, et al. Prevenção quaternária, reforma curricular e educação médica. Interface - comunicação, saúde, educação, 2020. 16: 4-12

6. DEPARTAMENTO DE INFORMÁTICA DO SISTEMA ÚNICO DE SAÚDE (DATASUS). 2017. Disponível em: http://tabnet.datasus.gov.br/cgi/tabcgi.exe?sim/cnv/ext10uf.def. Acessado em 9 de julho de 2020

7. GAMA MACG, et al. Os acidentes são evitáveis e na maioria das vezes, o perigo está dentro de casa!. Sociedade Brasileira de Pediatria, 2020. 9: 1-8

8. GURGEL AKC, MONTEIRO Al. Prevenção de acidentes domésticos infantis: susceptibilidade percebida pelas cuidadoras. Revista de Pesquisa Cuidado é Fundamental Online, 2016. 10: 1-9.

9. MACÊDO VC. Atenção integral à saúde da criança: políticas e indicadores de saúde. Recife: Ed. Universitária da UFPE, 2016. 43 p. Disponível em: https://ares.unasus.gov.br/acervo/html/ARES/9258/1/livro_saude_crianca.pdf. Acessado em 28 de maio de 2019

10. MENDES R, et al. Promoção da saúde e participação: abordagens e indagações. Saúde debate, 2016. 14: 2-11

11. MINISTÉRIO DA EDUCAÇÃO. Conselho Nacional da Educação. Diretrizes Curriculares Nacionais dos Cursos de Graduação em Enfermagem, Medicina e Nutrição. 2014. Disponível em: http://portal.mec.gov.br/cne/arquivos/pdf/2001/pces1133_01.pdf. Acessado em 20 de agosto de 2021. 
12. MINISTÉRIO DA SAÚDE. Portaria $n^{\circ} 1.130$, de 5 de agosto de 2015 . Disponível em: http://www.toledo.ufpr.br/portal/wp-content/uploads/2017/07/DCN-2014.pdf. Acessado em: 10 de outubro de 2021

13. MINISTÉRIO DA SAÚDE. Proteger e cuidar da saúde de adolescentes na Atenção Básica, 2017. Disponível em: https://bvsms.saude.gov.br/bvs/publicacoes/proteger_cuidar_adolescentes_atencao_basica.pdf. Acessado em 9 de julho de 2020

14. MINISTÉRIO DOS DIREITOS HUMANOS. Secretaria Nacional de Proteção dos Direitos da Criança e Adolescente. Violência contra Crianças e Adolescentes: Análise de Cenários e Propostas de Políticas Públicas, 2018. Disponível em: $\quad$ https://www.gov.br/mdh/pt-br/centrais-de-conteudo/crianca-e-adolescente/violencia-contra-criancas-eadolescentes-analise-de-cenarios-e-propostas-de-politicas-publicas-2.pdf. Acessado em 9 de julho de 2020.

15. NASCIMENTO EN, et al. Ações intersetoriais de prevenção de acidentes na educação infantil: opiniões do professor e conhecimentos dos alunos. Journal of Human Growth and Development, 2013

16. ORGANIZAÇÃO MUNDIAL DE SAÚDE. INSPIRE - Sete Estratégias para Pôr Fim à Violência Contra Crianças, 2016. Disponível em: https://apps.who.int/iris/bitstream/handle/10665/207717/9789241565356-por.pdf?ua=1. Acessado em 1 de agosto de 2021

17. REDE NACIONAL PRIMEIRA INFÂNCIA. Plano Nacional pela Primeira Infância 2010 - 2022 | 2020 - 2030. Brasília (DF), 2020. Disponível em: http://primeirainfancia.org.br/wp-content/uploads/2020/10/PNPI.pdf Acessado em: 10 de outubro de 2021.

18. ROMA KMS, et al. Accident prevention in early childhood in a family health strategy: parent's perspective. Revista de Enfermagem da Universidade Federal do Piauí, 2018. 7: 4-5

19. SENADO FEDERAL. [Constituição (2018)]. Estatuto da Criança e do Adolescente, 2017. Disponível em: https://www2.senado.leg.br/bdsf/bitstream/handle/id/534718/eca_1ed.pdf. Acessado em 10 de outubro de 2021

20. SOUZA RR, et al. A rede de atenção integral à saúde da criança no Distrito Federal, Brasil. Revista Ciência e Saúde Coletiva, 2019 